\title{
Design for Hydrogen Storage Materials via Observation of Adsorption Sites by Computer Tomography
}

\author{
Li Zhang Qi Wang* Ying-Chun Liu \\ Department of Chemistry, Zhejiang University, Hangzhou 310027, P. R. China
}

Supporting Information

The Computer Tomography for materials (mCT):

The mCT images for the simulation box, which consists of $2 \times 2 \times 2$ unit cells of MOF-5, were also produced and shown in Fig. S1. It is propitious to understand the adsorption sites of a MOF crystal from the viewpoint of whole based on the periodicity of the crystal.

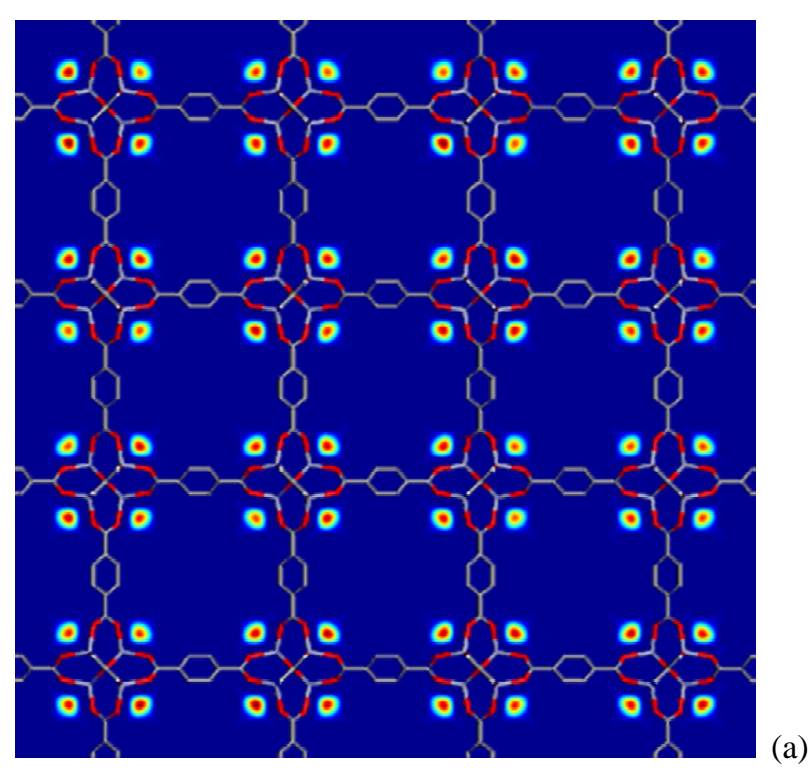



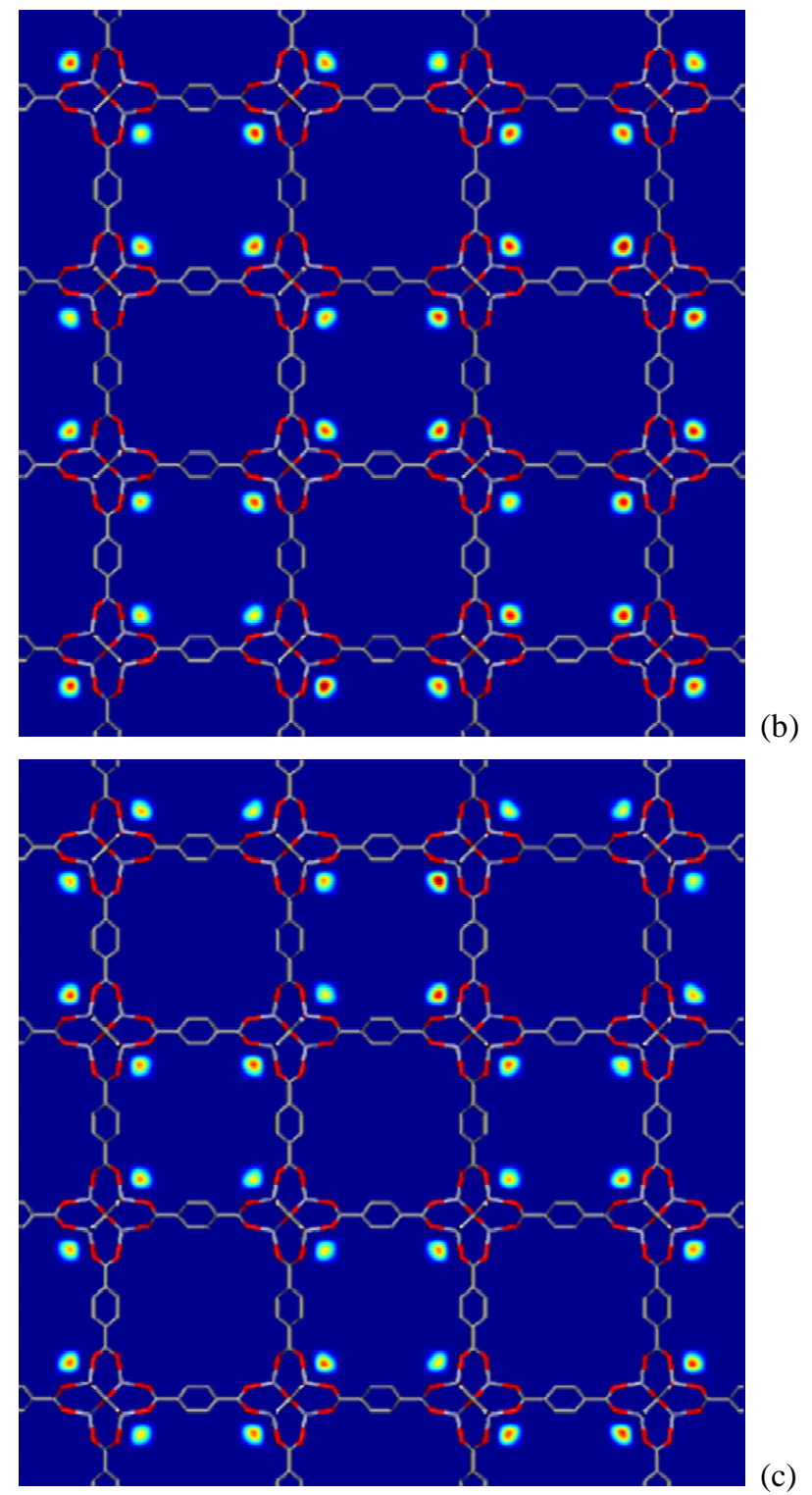


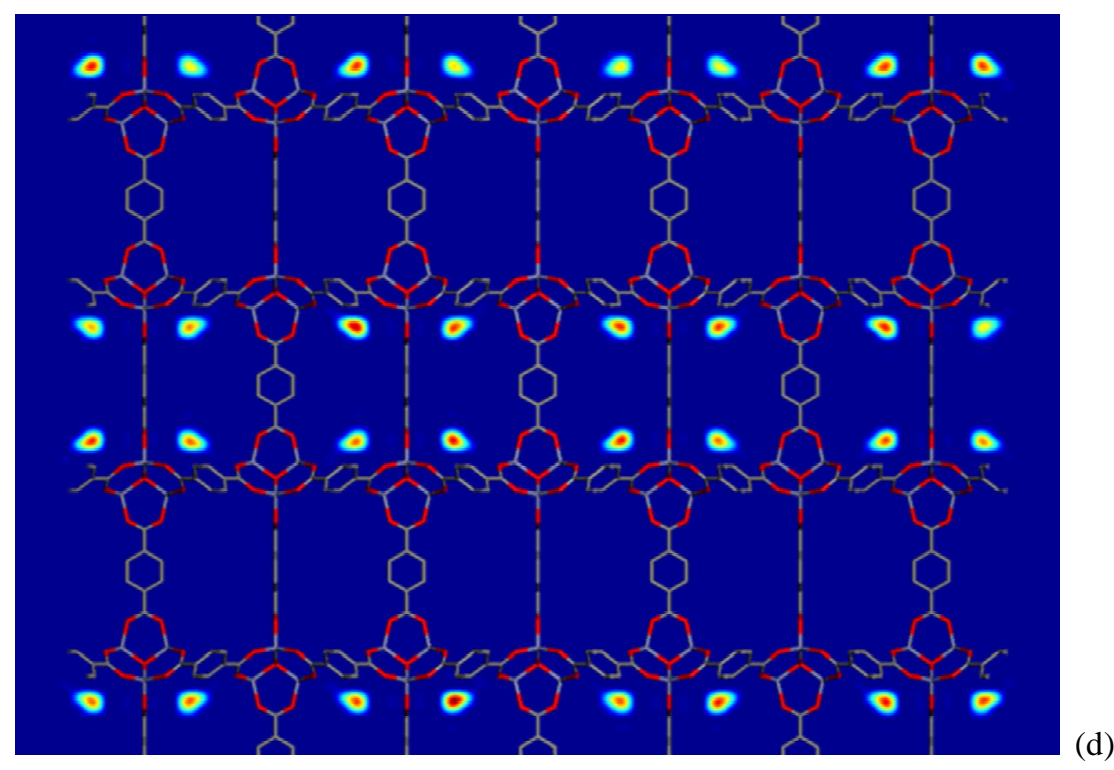

Fig. S1. mCT images for $2 \times 2 \times 2$ unit cells of MOF-5 at $30 \mathrm{~K}$, superimposed with the MOF-5 structure, indicating the location of cup sites for the first hydrogen adsorption (red-yellow-cyan region). (a) in the $x-y$ plane, the same in the $x-z$ and $y$ - $z$ planes, (b) in plane ' $A$ ', which is about $4.0 \AA$ in the $z$-direction, (c) in plane ' $\mathrm{B}$ ', about $9.0 \AA$ in the $z$-direction, and (d) in diagonal plane ' $\mathrm{C}$ ', the same in another diagonal plane ' $\mathrm{C}$ ', which is perpendicular to the plane ' $\mathrm{C}$ '. Similarly, the image is analogous to (d) for those in diagonal planes ' $D$ ' and ' $D$ ', just the adsorption sites are at the inner side of the horizontal frameworks.

The mCT images for hydrogen adsorbed in MOF-5 at $30 \mathrm{~K}$ and with loading of 4 molecules per unit cell were presented in the manuscript. It is in good agreement with the neutron powder diffraction experiments of Yildirim and Hartman, Fig. S2. However, the adsorption sites have not been reported at $77 \mathrm{~K}$ at which most of studies were performed by experiments and computations. In this work, the mCT images at $77 \mathrm{~K}$ and 1 bar were produced, Figs. S3-S6. It can be observed that the $\alpha(-\mathrm{COO})_{3}$ adsorption sites were also the primary adsorption sites as temperature increases up to $77 \mathrm{~K}$, and some secondary adsorption sites were observed close to the $\alpha(-\mathrm{COO})_{3}$ sites, which may be denoted as $\beta(-\mathrm{COO})_{3}$ as pointed out by Yaghi and co-workers. A few adsorbed molecules were found around the phenyl, it could be attributed to the higher loading of the molecules adsorbed (about $40 \mathrm{H}_{2}$ molecules per unit cell). The mCT images of the adsorbed $\mathrm{H}_{2}$ molecules in a cutting plane at the same positions as discussed in the manuscript were produced and shown in Figs. S4-S6.

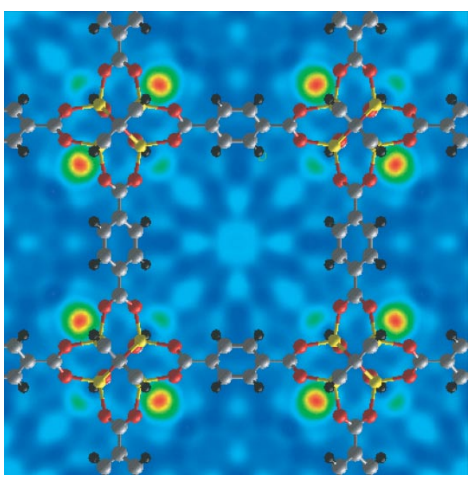


Fig. S2. (Fig. 3 in Ref. 9, for comparison) The neutron powder diffraction image ( $\lambda=2.08 \AA$ ) of the MOF-5- $4 \mathrm{D}_{2}$ at $3.5 \mathrm{~K}$. The real-space Fourier-difference scattering-length density superimposed with the MOF-5 structure, indicating the location of cup sites for the first hydrogen adsorption (red-yellow-green region).



Fig. S3. mCT image for $2 \times 2 \times 2$ unit cells of MOF-5 for all the hydrogen molecules adsorbed at $77 \mathrm{~K}$ and 1 bar in the $x-y$ plane, superimposed with the MOF-5 structure, indicating the location of the first-adsorption sites (red-yellow-cyan region) and the secondary adsorption sites (cyan-blue region), the same in the $x-z$ and $y-z$ planes.

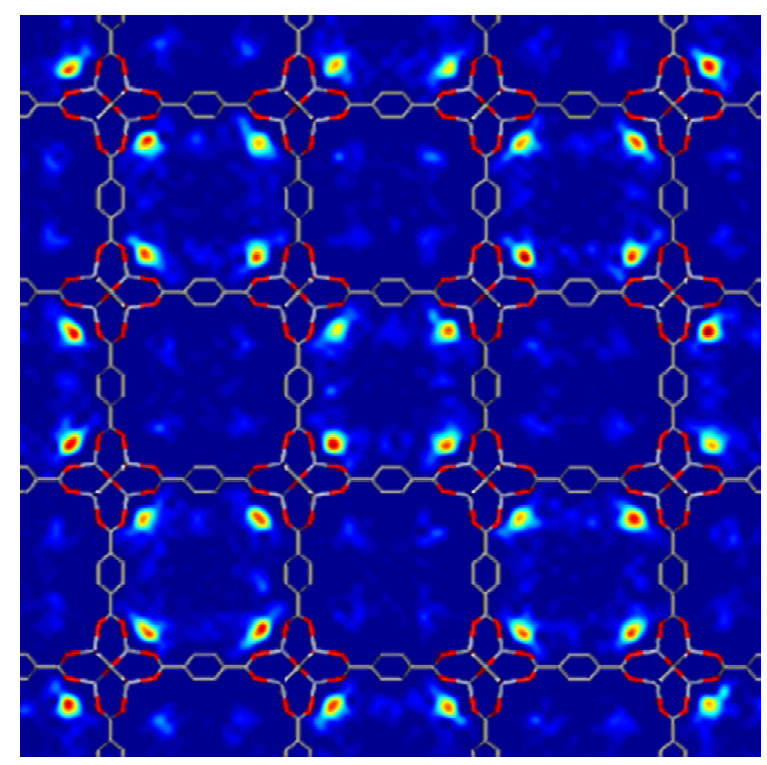

Fig. S4. mCT image for $2 \times 2 \times 2$ unit cells of MOF-5 for all the hydrogen molecules adsorbed at $77 \mathrm{~K}$ and 1 bar in plane ' $\mathrm{A}$ ', which is about $4.0 \AA$ in the $z$-direction. The blue region is the secondary adsorption site. 


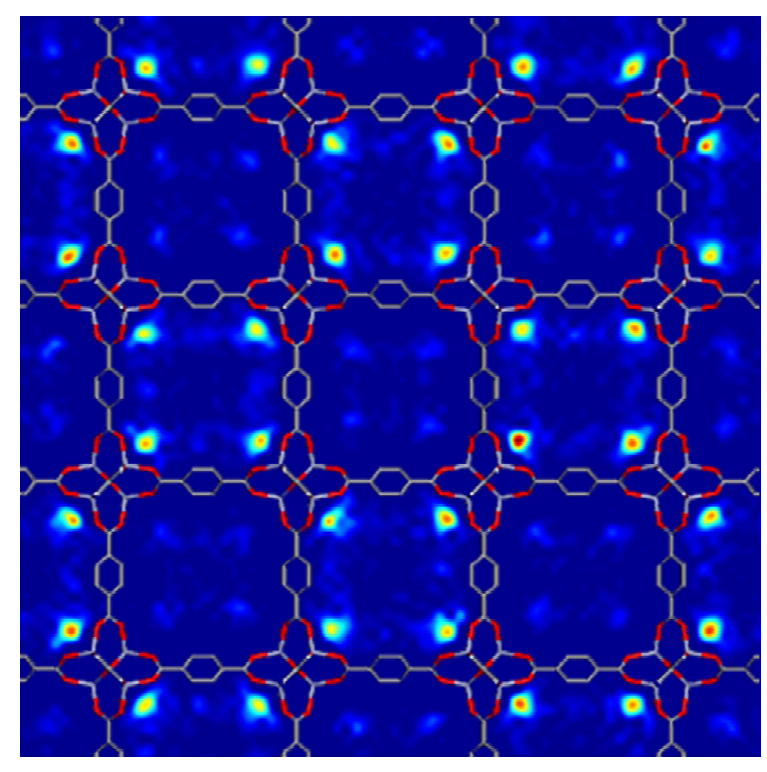

Fig. S5. mCT image for $2 \times 2 \times 2$ unit cells of MOF-5 for all the hydrogen molecules adsorbed at $77 \mathrm{~K}$ and 1 bar in plane ' $\mathrm{B}$ ', which is about $9.0 \AA$ in the $z$-direction. The blue region is the secondary adsorption site.

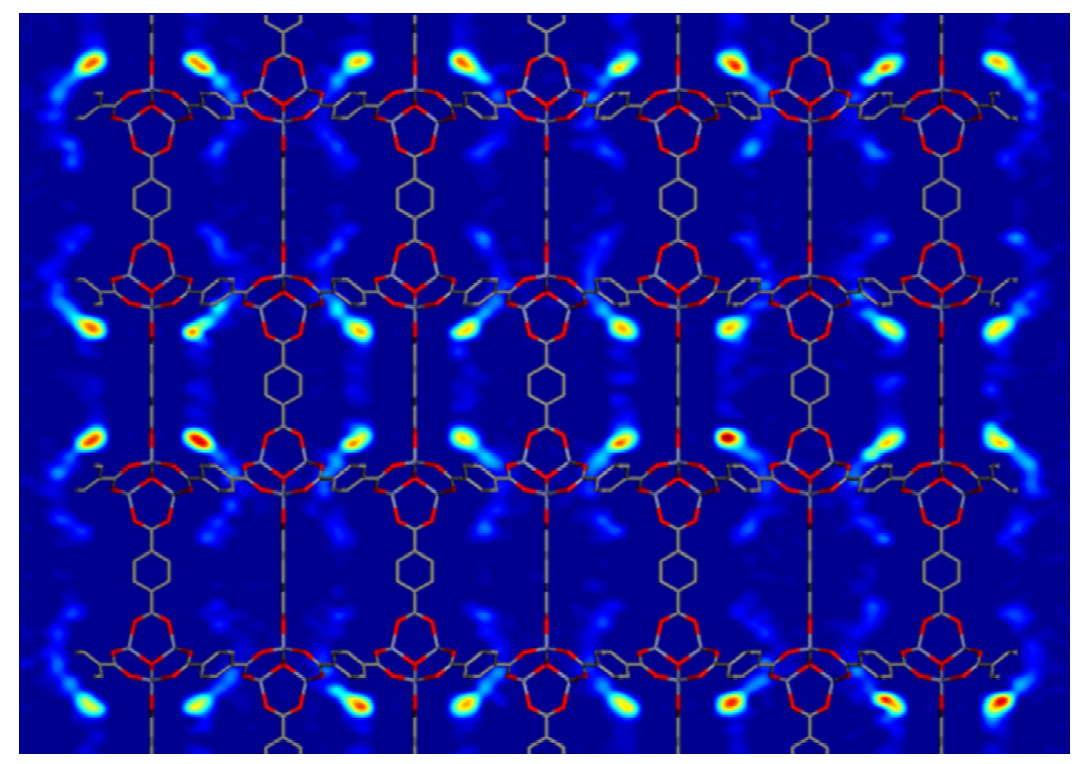

Fig. S6. mCT image for $2 \times 2 \times 2$ unit cells of MOF-5 for all the hydrogen molecules adsorbed at $77 \mathrm{~K}$ and 1 bar in diagonal plane ' $\mathrm{C}$ ', the same in another diagonal plane ' $\mathrm{C}$ ', which is perpendicular to the plane ' $\mathrm{C}$ '. The cyan-blue region is the secondary adsorption site. Similarly, the image is analogous for those in diagonal planes ' $\mathrm{D}$ ' and ' $\mathrm{D}$ ', just the adsorption sites are at the inner side of the horizontal frameworks. 